\title{
QUELQUES ASPECTS FONDAMENTAUX DU DEVELOPPEMENT JURIDIQUE ETHIOPIEN
}

\author{
Von JACQUeS VANDERLINDEN
}

Le problème du développement juridique de l'Ethiopie est généralement abordé sous l'angle des transformations du contenu du droit; ou encore sous celui de l'opposition entre droit traditionnel et droit moderne, voire entre une conception $\mathrm{du}$ droit le considérant comme un aboutissement du développement politique, économique et social et celle qui est en fait le moteur de ce développement; sous l'angle, pourrait-on dire enfin, de la distinction entre un droit sous-développé et un droit développé1. Mon propos n'est pas de gloser davantage sur ce thème rebattu auquel il devient difficile d'apporter de substantiels enrichissements. Je voudrais plutôt attirer l'attention sur certains aspects moins fréquemment traités et, à mon sens, tout aussi fondamentaux de ce problème. En effet, j'ai le sentiment que, si ces aspects ne sont pas pris en considération, bien des efforts entrepris pour moderniser le contenu du droit éthiopien risquent de demeurer vains.

Depuis plus de dix ans un courant de modernisation du droit s'est manifesté en Ethiopie; il a abouti à la promulgation de six codes (code civil, code de commerce, code maritime, code pénal, code de procédure civile et code de procédure pénale) dont le contenu trahit, dans l'écrasante majorité des cas, une option délibérée en faveur du droit moderne, du droit moteur du développement; cette orientation est confirmée dans les préfaces impériales qui présentent les textes au pays ${ }^{2}$. Et cependant, après quelques années d'application de ces codes modernes, on ne peut se retenir de penser que des problèmes fondamentaux relatifs à la formulation $\mathrm{du}$ droit, à sa diffusion et à sa mise en oeuvre effective auraient du être envisagés en même temps que s'effectuait la codification, permettant par là même un impact plus grand du droit nouveau sur la société éthiopienne. On constate en effet que cet impact est relativement réduit. Cette première considération entraîne, chez de nombreux juristes, un flot de commentaires, souvent ironiques, sur la vanité de vouloir imposer un droit moderne à des populations dont l'état de développement n'est de toute évidence pas au niveau des institutions juridiques destinées à gouverner leur vie quotidienne. On en revient ainsi à la grande querelle du droit traditionnel et du droit moderne en se refusant à essayer de réfléchir aux moyens qui permettraient éventuellement de faire du droit moderne un moteur du développement. Or, ces moyens semblent exister et c'est à leur examen que je voudrais consacrer ces quelques pages.

\footnotetext{
1 Parmi les études consacrées au développement juridique de l'Ethiopie, voir notamment celle de $R$. A. Sedler, The Development of Legal Systems: The Ethiopian Experience, Iowa Law Review, Vol. 53, 1967, pp. 562-635 qui est sans doute la plus récente et aussi parfaitement représentative de la tendance à centrer le début sur le contenu des règles juridiques.

2 Voir notamment la préface du Code pénal de 1957: "New concepts, not only judicial, but also those contributed by the sciences of sociology, psychology and, indeed penology, have been developed and must be taken into consideration in the elaboration of any criminal code . . ."; ou du Code civil de 1960: "The Civil Code has been promulgated by Us at a time when the progress achieved by Ethiopia requires the modernisation of the legal framework of Our Empire's social structure so as to keep peace with the changing circumstances of this world of today".
} 


\section{La formulation du droit}

Pour que le droit ait un impact sur la population, il convient qu'il soit formulé d'une manière qui soit accessible à celle-ci, c'est-à-dire dans un langage simple et cohérent qu'elle soit à même de comprendre. Ceci a été particulièrement bien exprimé dans la préface impériale au code civil éthiopien; l'empereur y déclare qu'il est essentiel que le droit soit clair et intelligible pour chacun des citoyens de son Empire de telle sorte qu'il puisse sans difficultés connaître ce que sont ses droits et obligations dans le cours quotidien de la vie ${ }^{3}$.

Un langage simple d'abord.

Lorsqu'on considère les développements du droit à travers l'histoire, on constate que les modes de formulation de celui-ci tendent à devenir de plus en plus sophistiqués au fur et à mesure que se développent les systèmes juridiques et la science du droit en particulier. Ainsi pouvons-nous comparer trois articles relatifs au vol jalonnant l'histoire juridique de l'Ethiopie, le premier emprunté au Fetha Nagast, le deuxième au Code pénal de 1930 et le troisième au Code pénal de 1957. En voici les textes:

- "Those who for the first time drive away cattle that do not belong to them shall be beaten; the second time, they shall be exiled; and on the third time, their hands shall be cut off. The cattle shall be returned to their owner"4.

- "A thief who by day steals any kind of property or cattle which are not his but belong to another man shall be imprisoned from one month to one year" 5 .

- "Whosoever, with intent to obtain or to procure to a third person an unlawful enrichment, abstracts a movable or a thing detached from an immovable, the property of another, whether by taking and carrying or by direct appropriation, or by having it passed indirectly to his own estate, is punishable ..." 6 .

Ce n'est là qu'un exemple qui pourrait être multiplié à l'infini. Il montre clairement qu'en se développant, le langage du droit devient de moins en moins accessible à ceux dont il doit en principe gouverner l'activité quotidienne. Cette constatation, qui se vérifie dans les pays développés, y présente cependant de moins graves inconvénients pour une double raison: d'une part la convergence fréquente entre l'état du droit et celui de la société fait que les membres de celle-ci vivent dans un cadre juridique qui leur est en général familier et qui fait ainsi partie intégrante de leur personnalité; d'autre part et surtout, l'existence d'une classe de juristes professionnels abondante et susceptible d'éclairer le non-spécialiste sur l'étendue de ses droits et obligations permet toujours (au moins à celui qui en a les moyens) de s'assurer de sa position face au droit.

Or, ce double facteur est précisément absent dans de nombreux pays en voie de développement et plus particulièrement en Ethiopie. D'une part, en vertu des principes mêmes qui président à son élaboration, le droit moderne introduit par la codification crée un cadre juridique souvent totalement différent de l'environnement social et économique dans lequel vivent les populations; d'autre part il n'existe pas de juristes professionnels qui soient susceptibles de les informer de

3 Civil Code of the Empire of Ethiopia, Addis Ababa 1960, p. VII.

4 The Fetha Nagast - The Law of the Kings, Addis Ababa 1968, p. 302.

5 The Ethiopian Penal Code of 1930, Addis Ababa [1941], p. 67.

6 Penal Code of the Empire of Ethiopia of 1957, Addis Ababa 1957, p. 190. 
manière valable sur le droit nouveau. Il est donc, dans ces conditions, encore plus important que le langage de la loi soit aussi simple que possible de manière à pouvoir être compris du plus grand nombre de citoyens.

Si nous reprenons l'exemple précité de la définition du vol, on peut se demander quel est l'objectif poursuivi par le rédacteur du Code en proposant une définition aussi complexe et en ayant recours à des termes comme "third party", "unlawful enrichment", "abstracts" or "appropriation"? La réponse semble aller de soi: le souci de précision de manière à ce qu'aucune ambiguïté soit possible. Mais ce désir d'éviter l'ambiguïté n'aboutit-il pas en fait à la créer dans l'esprit non seulement de plus de 20.000 .000 de justiciables, mais encore dans celui d'une large fraction des magistrats, lesquels n'ont pas été formés au droit nouveau? Il est raisonnable de croire, et la pratique le montre souvent, qu'aussi bien les justiciables que de nombreux magistrats s'arrêteront au seul mot "vol", qui figure dans le Code en tête de l'article immédiatement après son numéro d'ordre, sans se soucier de vérifier si les faits de la cause constituent effectivement "la soustraction d'une chose mobilière ou d'une chose détachée d'un immeuble appartenant à autrui, dans le dessein de procurer à un tiers un enrichissement illégitime, que ce soit en l'enlevant ou en se l'appropriant de manière directe, ou en le faisant passer indirectement dans son patrimoine" 7 . Ceci est d'autant plus intéressant à constater que le rédacteur du Code estime avoir utilisé "des dispositions brèves, frappées le plus possible en maximes", formulée d'une manière "facile à saisir et pour ainsi dire populaire"; on ne peut s'empêcher d'être frappé par l'écart existant entre le désir extrêmement louable et la réalité. Par contre il faut reconnaître que l'opération n'est pas aisée en toutes circonstances. Il n'empêche qu'un effort systématique dans le sens de la simplicité d'expression paraît capital sans qu'il doive nuire pour autant à la qualité du contenu des règles exprimées.

Un langage cohérent ensuite.

En Ethiopie se développe pour l'instant de manière absolument empirique et inconsistante une langue jusqu'à présent peu adaptée aux concepts juridiques modernes. Cette langue, consacrée comme la seule langue nationale de l'Empire, est l'amharique; en cela, l'Ethiopie ressemble à des pays en voie de développement comme le Burundi, Madagascar, le Rwanda ou la Tanzanie qui se sont donnés, depuis leur indépendance, une langue nationale qui ne soit pas une langue européenne ou l'arabe. C'est évidemment dans ces langues que l'écrasante majorité de l'activité juridique du pays se déroule et, par conséquent, il est de première importance que l'outil ainsi utilisé soit le plus consistant possible. Or, il faut bien constater que c'est loin d'être le cas; la législation moderne trahit une propension à ne pas utiliser le terme le plus adéquat, à utiliser des termes différents pour désigner des réalités identiques ou des termes identiques pour désigner des réalités différentes. Il en résulte d'innombrables confusions qui ne peuvent que desservir l'efficacité du droit moderne.

Il faut même reconnaître que, dans certains cas, heureusement peu nombreux, les experts ayant rédigé les codes ont encouragé cette tendance. Ainsi, pour prendre maintenant les exemples dans le champs du droit civil, le professeur David a-t-il occasionellement accru les confusions possibles par un certain manque de rigueur dans la rédaction de son Code. Le mot "succession" est employé soit pour désigner le processus par lequel les droits et obligations d'une personne décédée sont

7 Extrait adapté de Le Code pénal de l'Empire d'Ethiopie (du 23 juillet 1957), Paris 1959, p. 167. 
transmis à ses héritiers, soit l'ensemble de ces droits et obligations que le rédacteur appelle par ailleurs "l'hérédité". De même l'utilisation de mots qui ont, dans la langue du rédacteur, une signification précise et auxquels il confère un sens nouveau, est susceptible de créer des confusions. Ainsi le "patronyme", qui est, en doctrine française, l'équivalent du "nom de famille" est utilisé dans le Code pour signifier le nom du père, ce qui est sans doute plus conforme à l'étymologie du mot, mais risque à nouveau de créer des confusions dès que l'Ethiopien sera amené à se référer à une autre langue que la sienne ${ }^{9}$.

Sur le plan de la consécration de termes amhariques courants dans la langue juridique, un point est particulièrement important: ne pas trop s'éloigner de la signification usuelle et surtout éviter une interversion de termes pouvant induire aisément à certaines confusions. Ainsi les mots "assadari" et "mogzit" employés respectivement dans le Code civil pour désigner le gouverneur et le tuteur du mineur semblent, dans la langue usuelle et en tout cas en ce qui concerne le second, correspondre exactement aux réalités inverses. Le premier est parfois utilisé pour désigner le chef de famille au sens large en tant que responsable du groupe qu'il dirige ou bien la personne qui pourvoit à la subsistence matérielle d'une autre, tandis que le second se réfère à des activités éducatrices du genre de celles confiées en Europe à une gouvernante. En outre, il faut remarquer que le langage du Code s'éloigne ici également de celui, traditionnel, du Fetha Nagast qui consacre "mogzit" pour gouverneur et "assadagi" pour tuteur.

Une priorité doit donc être donnée à la constitution d'un vocabulaire juridique précis et surtout cohérent. La Faculté de Droit de l'Université Haile Selassie I s'est rapidement rendue compte du caractère impératif de ce problème pour le développement juridique de l'Ethiopie et il est regrettable de constater que tous ses efforts pour voir soutenir financièrement son projet d'un lexique juridique amharique-anglais-français se sont heurtés à des fins de non-recevoir de la part des organismes bailleurs de fonds qu'ils soient nationaux ou internationaux ${ }^{\mathbf{1 0}}$.

Un langage intelligible pour tous enfin.

La langue officielle de l'Empire éthiopien est, nous l'avons vu, l'amharique; par une tolérance qui remonte à la libération du pays par les troupes anglaises et à l'établissement du premier véritable journal officiell, les textes législatifs sont également publiés en anglais. C'est là un effort de courtoisie considérable destiné à assurer la connaissance du droit national par l'importante colonie étrangère vivant dans le pays et jouant un rôle actif dans son développement économique et social. Mais que penser de l'impact d'un journal officiel rédigé en amharique et en anglais sur les populations, et elles sont nombreuses, qui n'entendent ni l'une ni l'autre de ces langues? Pour elles, le droit nouveau doit nécessairement rester lettre morte. $\mathrm{Si}$ on envisage les précédents historiques, on trouve, dans l'histoire d'Ethiopie des exemples d'une attitude fort différente, puisque les proclamations de Ménélik dans les régions où l'amharique n'était pas la langue dominante, étaient diffusées aussi bien dans cette langue que dans les langues locales. Ainsi, savons-nous qu'en 1908, les proclamations étaient faites à Dire Dawa, en amharique, harari, gallinia et somali11; cette méthode permettait certainement aux diverses populations de

8 Voir Code civil de l'Empire d'Ethiopie de 1960, Paris 1962, par exemple Art. 826 (2) où est définie l'hérédité et Art. 942 où la même notion est qualifiée de "succession", alors que l'ensemble du Titre V du Livre II utilise le mot "succession" pour designer le mécanisme successoral dans son ensemble.

9 Voir Idem, Art. 32 et 36.

10 Voir sur le projet de Lexicon, l'article de S. Z. Fisher et Fassil Abebe dans le Journal of Ethiopian Law, vol. V, 1958, p. 553.

11 Le Semeur d'Ethiopie, Novembre 1908, p. 493. 
l'Empire de mieux connaître le contenu de la volonté impériale. Il semble toutefois que cette pratique ait disparu avec Ménélik et n'ait plus été suivie au cours des années suivant la première guerre mondiale. Il existe certes des justifications politiques à l'attitude consistant à ne publier les lois qu'en une seule langue, celle reconnue comme nationale par la Constitution; la plus importante est sans doute relative à l'unité du pays qui est grandement encouragée par l'unité linguistique. Il n'y a aucun doute à cet égard, mais il n'en demeure pas moins qu'un droit formulé de manière totalement incompréhensible pour les habitants ne peut prétendre être aussi le moteur d'un changement dans leur comportement quotidien. D'autres problèmes, d'ordre technique ceux-là, peuvent être soulevés, comme ceux relatifs à la traduction dans un nombre important de langues mal connues ou celui d'éventuelles discordances entre les diverses versions des textes; mais ils sont loin d'être insolubles et de nombreux pays multilingues nous offrent des exemples susceptibles d'inspirer des solutions satisfaisantes à la fois pour les intéressés et pour le gouvernement.

Tels sont les trois points qui attirent le plus l'attention en ce qui concerne la formulation du droit. Ils constituent la base de départ sur laquelle tous les développements qui suivent sont bâtis.

\section{La diffusion du droit}

A supposer que le droit moderne soit accessible aux populations éthiopiennes grâce à un langage simple, cohérent et compréhensible, il faut encore que sa diffusion au sein de la population soit assurée.

De noveau on ne peut manquer d'être frappé par les enseignements de l'histoire du droit éthiopien. Celle-ci nous montre en effet que l'on est passé progressivement d'un droit dont la diffusion était assurée au maximum parmi les populations à un droit qu'il devient de plus en plus difficile de connaître. Autrefois, le droit était proclamé dans les villes et villages selon un cérémonial bien défini. Dans la capitale, une porte de l'enceinte du palais impérial possédait un balcon surélevé, sur lequel venait s'installer, selon un protocole minutieusement établi, de nombreux dignitaires, tandis que le Ministre de la Plume proclamait au peuple assemblé la volonté de l'Empereur. De même, en province, les hérauts impériaux procédaient à la lecture publique des proclamations après que le tambour ait résonné pour convier la population à l'entendre et que le symbole de la monarchie, en l'occurrence très souvent un lion, ait été présenté au public ${ }^{12}$. Dès le règne de Ménélik cependant, les premières traces du modernisme se faisaient sentir avec l'impression des premières proclamations ${ }^{13}$. La pratique se développa et le pas suivant fut la création d'un journal semi-officiel par le Ras Taffari, le future Empereur Haile Selassie Ier, en 1924; intitulé "Lumière et Paix", le périodique contient notamment une grande partie de la législation promulguée entre 1924 et 1935 et inaugure le bilinguisme officiel en reproduisant certains textes, intéressant plus particulière-

\footnotetext{
12 Ibidem et aussi, pour des périodes antérieures, C. Conti Rossini, "Sue due frasi della Cronaca abbreviata dei Re d'Ethiopia", Annali dell'Istituto orientale di Napoli, Vol. III, 1949, pp. 284-290. Pour les périodes récentes, voir notamment la règlementation des proclamations dans les Zikire Negar, du Balambaras Mahteme Selassie Wolde Meskel (Addis Ababa 1951), dont la couverture reproduit une scène de proclamation telle que se la rappelle l'auteur. Certains éthiopiens m'ont assuré que la Constitution revisée de 1955 avait encore été proclamée sur certaines places publiques de la capitale.

13 Voir notamment $H$. d'Orleans, Une visite à l'Empereur Menelik, Paris 1898, p. 194, qui relate la première impression d'une proclamation par Mondon-Vidailhet en 1897.
} 
ment les étrangers, en français. A ce moment, il ne restait plus qu'un pas à franchir et ce fut chose faite en 1942, lors de la libération du pays: la Negarit Gazeta est créée, qui publie officiellement en amharique et en anglais, toute législation applicable dans l'Empire. Simultanément la pratique des proclamations publiques disparaît. Aujourd'hui la diffusion des lois se fait par le seul journal officiel et il est permis de s'interroger sur son efficacité en tant qu'organe de diffusion de la loi. Dans un pays où le taux d'analphabétisme demeure considérable, il semble que la grande masse des justiciables doive demeurer dans une ignorance presque totale de la loi si celle-ci ne lui est accessible qu'à travers l'imprimé. La connaissance du droit, et plus particulièrement du droit moderne qui s'écarte tellement du cadre social et économique traditionnel, risque en conséquence d'être faible sinon nulle, et l'impact des institutiones nouvelles sur la population tout aussi faible, voire nul. On peut d'ailleurs se demander si le problème de la diffusion ne doit pas être entièrement repensé en tenant compte des larges possibilités qu'offrent aujourd'hui les techniques de diffusion collective; voire même des moyen traditionnels et moins sophistiqués dont l'impact risque d'être plus grand que la proclamation traditionnelle aussi solennelle soit-elle.

Considérons d'abord les techniques susceptibles d'être utilisées. Parmi les techniques que je qualifierais volontiers de populaires, il faut ranger le recours à l'affiche, au théâtre, à la chanson, à la presse, à la littérature. Ce sont là en effet toutes espèces de moyens de communication dont l'impact est certes variable, mais dont l'efficacité serait indéniablement plus grande que la seule publication au journal officiel. Je voudrais d'ailleurs particulièrement insister ici sur les techniques audio-visuelles qui font uniquement appel à l'oreille et à la vue des citoyens étant donné qu'elles sont les seules vraiment efficaces dans un pays où le taux d'analphabétisme reste aussi important. Il est certain qu'une saynète comique ou une chanson humoristique mettant en scène les malheurs de celui qui a choisi d'ignorer le Code civil pour lui préférer la tradition sera bien plus susceptible d'encourager un intérêt pour le droit moderne, voire un changement d'attitude, que des tonnes d'imprimés traitant $\mathrm{du}$ même thème sur un ton plus austère. La même chose est vraie en ce qui concerne le dessin animé dont certains expériences dans le domaine commercial ont pu montrer l'impact qu'il avait sur les masses africaines; la Barclays Bank a vu se multiplier ses clients en Afrique occidentale anglaise après la diffusion sur les écrans de la région d'un dessin animé montrant de façon pittoresque les avantages de la banque en matière économique. De nouveau, certains juristes trouveront sans doute indigne d'un monument de la science juridique comparée comme le Code civil qu'il soit porté à l'attention de ceux qu'il régit sous des formes aussi peu austères; je leur répondrai qu'il vaut mieux un code efficace à travers le dessin animé ou la bande dessinée qu'un code inopérant enfermé dans la dignité d'un journal officiel.

Il va d'ailleurs de soi qu'à côté de ces techniques dites populaires il en existe d'innombrables autres qui sont susceptibles de contribuer à ce qu'on pourrait appeler la vulgarisation du droit. Entre l'habitant analphabète des régions les plus reculées de l'Empire et le juge à la Cour suprême titulaire d'un diplôme de licencié, voire de docteur en droit, il existe une infinie gradation dans le développement intellectuel et les capacités d'appréhension du phénomène juridique. Il importe donc que le droit puisse être diffusé en tenant compte de cette gradation selon des techniques appropriées et adaptées au niveau du développement des diverses classes sociales formant la population. L'essentiel est l'efficacité de la méthode utilisée en 
ce qu'elle rendra le droit moderne accessible à la plus grande masse possible de citoyens. Certains pays africains ont d'ailleurs ouvert la voie en ce sens. Je voudrais seulement mentionner, à titre d'exemple, la République malgache qui soutient les efforts de l'Ecole nationale de Promotion sociale, laquelle publie des "Guides d'initiation active au développement" dont un volume est consacré aux "Institutions politiques et administratives de la République malgache". Ces guides s'adressent à un public éduqué, mais non-juriste, et mettent à sa disposition de manière claire et imagée les problèmes essentiels du développement ${ }^{14}$.

Une fois définies les techniques de diffusion en fonction du public qu'elles doivent atteindre, le problème du moment et de l'endroit où les utiliser est posé. De nouveau les considérations d'efficacité doivent primer dans ce cas et les techniques doivent par conséquent être utilisées partout où elles sont susceptibles de porter des fruits.

On pense ainsi immédiatement à l'école où se forment les générations qui construiront l'Ethiopie de demain. Non seulement les jeunes sont souvent plus malléables que leurs aînés, mais aussi ils sont souvent animés d'un désir de progrès dont le droit nouveau est précisément le symbole. Il y a donc là un terrain tout indiqué pour semer la graine du développement, ne serait-ce que de façon rudimentaire; l'essentiel est que la prise de conscience de l'existence d'une rupture avec la tradition juridique s'implante aussi rapidement que possible dans l'esprit de la jeunesse. Mais les adultes aussi doivent être touchés partout où il est possible de les atteindre. Dans les milieux urbains la radiodiffusion, voire la télévision, constituent des moyens de premier ordre pour faire pénétrer les idées nouvelles. Une initiative en ce sens a été lancée sur les ondes de la radio éthiopienne par la Faculté de Droit de l'Université d'Addis Abéba. Elle consiste en une série d'émissions intitulées "Vous êtes le juge" au cours desquelles un problème juridique est présenté aux auditeurs, des solutions sont défendues par des étudiants pour chacune des parties en présence et la solution sera finalement proposée par les auditeurs. Au cours d'une émission ultérieure, la critique des réponses et l'exposé de la solution correcte sont présentés au public. Malheureusement la radiodiffusion ne touche guère les milieux ruraux. Il importe donc de les atteindre autrement et notamment en tirant parti de toute espèce d'efforts accomplis dans leur direction. Il est souvent question, dans les pays en voie de développement, d'animation rurale. Pourquoi ne pas faire l'animation juridique à l'occasion d'autres formes d'animation et ne pas inclure le droit nouveau dans tout programme tendant au développement économique ou social de la masse? Il convient évidemment que la contribution juridique à ces programmes soit efficace et se situe donc au niveau du développement des populations en cause; mais cela est également vrai des techniques générales d'animation dont l'animation juridique ne constitue qu'une partie.

En conclusion, des solutions au problème de la diffusion existent; il importe seulement de les adapter à la diffusion du droit. Cette adaptation requiert certainement de l'imagination et des ressources financièrs et sans doute davantage de la première que des secondes. L'inconvénient est que les juristes manquent souvent de cette première qualité. Elevés dans une tradition, qu'elle soit continentale ou anglo-saxonne, ils ont des difficultés à comprendre la réalité complexe du continent africain et se contentent en conséquence le plus souvent de construire des mécanismes dont les aspects techniques sont le fruit des connaissances les plus développées,

14 L'auteur en est M. Massiot (Tananarive 1967). 
mais dont l'adéquacité est souvent douteuse dans le contexte particulier où ils sont plongés. Sociologues et psychologues sont certainement mieux à même de réaliser cette nécessaire adaptation des techniques du monde dit "développé” à celui considéré comme "sous développé". Malheureusement, il leur manque la formation juridique, qui est tout aussi essentielle. Le champ est donc ouvert à l'activité interdisciplinaire ou à cette race nouvelle de juristes dont il existe à l'heure actuelle trop peu de spécimes: les juristes du développement.

\section{La mise en oeuvre effective du droit}

Si une formulation satisfaisante et une diffusion adéquate du droit sont des exigences fondamentales du développement juridique, la mise en oeuvre du droit nouveau est également, sinon plus importante. En effet, peu importe que le citoyen connaisse effectivement ses droits et obligations, si, au moment où il en demande la reconnaissance devant le juge, celui-ci s'avère incapable de la lui fournir ou, pis encore, si le citoyen se trouve confronté, au sein même de sa société à une opposition, latente et toléreé par le gouvernement, au droit moderne. Si la première hypothèse risque de détruire la confiance du citoyen dans les autorités judiciaires, la seconde peut avoir l'effet bien plus grave encore d'ébranler sa foi en la législation en tant que symbole de la volonté étatique de progrès.

Au moment où le Code civil éthiopien fut promulgué, en 1960, le pays ne comptait sans doute pas plus qu'une poignée de juristes formés aux techniques du droit moderne; ceux-ci étaient, en majorité, des étrangers, de nationalités diverses et ils occupaient des postes-clés de l'administration de la justice, comme la présidence de la High Court ou des sièges, voire la présidence occasionnelle de certaines chambres de la Cour Suprême: de juristes éthiopiens titulaires d'un diplôme couronnant des études juridiques, il n'y en eut pas avant quelques années, lorsqu'un premier contingent, formé en grande partie au Canada, vint étoffer les cadres de la fonction judiciaire nationale. Ceux-ci demeuraient néanmoins une minorité face à l'écrasante majorité des juges non formés, pour lesquels un certain bon sens devait, dans le plus grand nombre des cas, suppléer aux lacunes de leur éducation juridique. Dans ces conditions, il n'est guère étonnant que certaines décisions, rendues en des matières importantes en ce qui concerne la vie quotidienne des individus, fassent preuve d'une incompréhension totale de l'esprit dans lequel le droit moderne a été conçu en écartant délibérément certaines de ses dispositions parmi les plus claires; je me limiterai à deux exemples caractéristiques.

Le premier concerne une affaire de succession et la validité des testaments. L'article 881 (2) du Code civil prévoit que le testament public “à peine de nullité, ... est lu en présence du testateur et de quatre témoins et porte mention de l'accomplissement de cette formalité et de sa date"; voilà sans doute un des meilleurs exemples de disposition qui ne prête guère à ambiguïté. Or, la Cour Suprême, confrontée avec un testament dont l'élaboration n'avait en lieu qu'en présence de trois témoins, décida, malgré l'avis de la Haute Cour en première instance, d'en affirmer la validité. Ecartant la motivation du jugement dont appel fut fait, lequel constatait à bon droit que "les règles relatives à la forme des testaments ont été promulguées de manière à s'assurer que seule l'intention indubitable du testateur produit des effets juridiques et désigne comme successeurs d'autres personnes que ses héritiers ab intestat", que "de telles règles doivent être strictement interprétées, 
la Cour ne pouvant, même dans des circonstances comme celles de l'affaire en cause, les ignorer ou y apporter des accomodements", la Cour Suprême estima que "dans des affaires où le tribunal de première instance sait que l'intention présumée du défunt est de faire de son épouse son successeur à l'égard de la totalité de ses biens, il ne doit pas se laisser aveugler par des questions de forme . .." et que "l'absence d'un témoin, lorsque trois d'entre eux sont présents, est sans importance"15. J'ai, dans une étude précédente ${ }^{16}$, défendu le point de vue qu'une certaine flexibilité dans l'interprétation était sans doute indispensable à l'élaboration d'un droit moderne dans les pays en voie de développement; l'exemple que je citais était précisément celui-ci, qui était, à ce moment l'un des seuls disponibles. Je ne crois pas devoir fondamentalement altérer ma prise de position d'il y a quelques années; mais je dois à la vérité de reconnaître que, dans des cas aussi clairs, où aucune considération relative à une certaine ambiguïté du droit n'est en cause, l'ignorance délibérée du prescrit légal par le juge ne peut être que source de confusions dans l'esprit des justiciables.

La seconde affaire concerne une matière tout aussi importante dans la vie courante des Ethiopiens: le mariage et l'incidence du mariage religieux sur la dissolution du mariage civil. De nouveau il semble que le Code ait pris une position nette à cet égard. L'article $662 \mathrm{du}$ Code civil commence par définir l'égalité totale des diverses formes (civil, religieux ou coutumier) du mariage devant les règles de dissolution. Il le fait sans aucune ambiguïté et même de manière répétitive en déclarant: "(1) Les causes de dissolution du mariage et les effets de cette dissolution sont les mêmes, quelle que soit la forme selon laquelle le mariage a été célébré.” “(2) Il n'y a pas lieu de distinguer, à ce sujet, selon que le mariage a été célébré devant un officier de l'état civil ou selon qu'il a été célébré suivant les formes prescrites par la religion ou la coutume". En outre, l'article 663 énumère limitativement les causes possibles de dissolution du lien conjugal. Ce sont la mort d'un des époux, le prononcé de la dissolution par les juges à titre de sanction d'une des conditions du mariage, et le divorce. Enfin l'article 671 du Code prévoit que la dissolution du mariage religieux par les autorités ecclésiastiques peut être considérée comme une cause grave de divorce civil aux yeux de la loi éthiopienne. C'est là la seule mention du mariage religieux dans les articles relatifs à la dissolution du mariage, si on excepte, celui déjà cité, qui établit l'égalité complète des diverses formes de mariage aux yeux du droit moderne du divorce. La Cour Suprême, confrontée avec une demande de dissolution d'un mariage conclu à l'église, alors que ce mariage religieux n'avait pas été annulé par les autorités religieuses, refusa le divorce estimant que la dissolution du mariage religieux était une condition nécessaire préalable à toute décision prise par les juridictions civiles. De nouveau, il est clair qu'il y a incompréhension caractérisée du prescrit légal. D'une part, le Code dit clairement que la forme sous laquelle le mariage a été célébré est sans importance en ce qui concerne le divorce; de l'autre, l'article 671 dit qu'il y a cause grave aux yeux de la loi sur le divorce si le mariage religieux a été annulé. Il n'est donc aucunement question de conférer aux autorités ecclésiastiques un droit de contrôle sur la dissolution des mariages religieux dans la mesure où l'activité des juridictions civiles est en cause. Il n'empêche que la Cour Suprême a cru pouvoir imposer cette "interprétation" pour autant qu'on puisse encore parler d'interpré-

15 Journal of Ethiopian Law, vol. 1, 1964, pp. 26-29 and 32-33.

16 "Civil law and common law influences on the developing law of Ethiopia", Buffalo Law Review, Vol. 16, 1966, pp. 230-266. 
tation en l'occurence ${ }^{17}$. De nouveau le degré de formation juridique des juges est clairement en cause et, comme dans le cas précédent, la clarté et la diffusion du droit sont sans défense devant sa mise en oeuvre erronée.

Ceci est d'autant plus grave qu'il ne s'agit pas de juridictions d'un niveau inférieur, mais bien de la plus haute Cour du pays (à l'exception de la cour privée de l'Empereur, laquelle ne siège qu'en équité). Il faut en outre signaler qu'il existe une Proclamation sur l'administration de la Justice, actuellement suspendue ${ }^{18}$, dont un des articles prévoit que les décisions des cours supérieures lieront les cours inférieures; on mesure ainsi toute l'importance des décisions de la Cour Suprême. A cet égard d'ailleurs, il est permis de se demander s'il est sage, aussi longtemps que le niveau de formation des magistrats demeurera aussi rudimentaire, de conférer aux décisions d'un tribunal, aussi haut placé soit-il, une autorité aussi absolue sur les juridictions qui lui sont inférieures. On risque en effet d'accréditer dans le public des "interprétations" de la loi du genre de celles que nous venons de mentionner et les effets sur l'application effective de la législation risquent d'en être désastreux. Ceci bien entendu lorsqu'il s'agit de retourner complètement un prescrit législatif parfaitement clair; la situation est toute autre lorsqu'il s'agit de trancher des difficultés d'interprétation ou des discordances entre articles ou entre versions différentes du même texte. Dans ces cas, le rôle des juridictions et même une certaine unité d'interprétation, sont capitales. Mais alors comment distinguer l'un cas de l'autre et éviter que la Cour Suprême, pour ne prendre que cet exemple, ne tranche aussi bien contre la loi qu'en sa faveur. A tout prendre, je serais d'avis qu'il vaut mieux encore éviter le caractère contraignant du précédent judiciaire, plutôt que de laisser s'accréditer des opinions aussi manifestement contraires au voeu du législateur. En tout état de cause, la situation n'est que temporaire et le relèvement du niveau d'éducation des magistrats y remédiera progressivement.

Le problème de la mise en oeuvre effective du droit au niveau de la Cour Suprême, s'il présente une certaine importance au niveau de l'affirmation du droit moderne sur le plan des principes, n'a évidemment pas l'impact sur la population que peut présenter la même mise en oeuvre effectuée par les juridictions inférieures au niveau des populations. Après ce qui a été dit des juridictions supérieures, il est sans doute inutile d'insister sur ce que peut être l'application du droit au niveau des villages de province, ceci d'autant plus que la mise en vigueur du droit nouveau n'a pas pour autant fait disparaître de l'organisation judiciaire, du moins pendant un certain temps (en fait pendant 5 ans jusqu'à la promulgation du Code de Procédure civile), les juges traditionnels décidant en fonction des usages locaux. En principe, sans doute, ceux-ci devaient administrer le Code; on voit aisément où pareille attitude théorique risquait de conduire dans la pratique. Le Code de Procédure civile de 1965 remédie partiellement à cet inconvénient en faisant disparaître les juridictions traditionnelles; le problème de l'éducation juridique des juges de niveau inférieur n'en demeure pas moins posé. Malgré un vaste effort de la Faculté de Droit de l'Université Haile Selassie en vue de fournir ne serait-ce qu'une teinture du droit moderne à des centaines de praticiens grâce à des programmes spéciaux conférant des "certificats", il n'en demeure pas moins que l'influence éducative ne dépasse guère le cadre des grands centres et est tellement superficielle et temporaire qu'il est permis de se demander quels sont ses effets à long terme

17 Journal of Ethiopian Law, vol. 3, 1966, pp. 390-398 et la current issue par N. Singer, "The dissolution of religious marriages in Ethiopia", idem, vol. 4, 1967, pp. 205-210.

18 Voir Negarit Gazeta, Vol. 22, 1962, pp. 70-75. 
sur la mise en application efficace du droit moderne. Selon toute vraisemblance, il faudra encore plusieurs années (pour ne pas compter en décennies) pour que celui-ci soit assuré d'une application satisfaisante dans tout l'Empire.

Le second aspect majeur de la mise en oeuvre effective du droit concerne l'opposition délibérée que celu-ci peut rencontrer dans toutes les couches de la population, y compris celles qui ont le niveau d'éducation requis pour apprécier la raison d'être du droit nouveau. On rencontre dans ce cas un refus généralisé d'application de la loi, refus devant lequel l'autorité judiciaire, voire politique, ne réagit pas. L'exemple le plus caractéristique en est sans doute la matière du nom dans le Code civil. En vertu de l'Article $32 \mathrm{du}$ Code, toute personne possède un nom de famille, un ou plusieurs prénoms et un patronyme (ce dernier est le prénom usuel du père); si le ou les prénoms, tout comme le patronyme, font partie de la tradition éthiopienne, il en va tout autrement du nom de famille qui est une innovation du Code introduite vraisemblablement afin de faciliter l'identification et de renforcer le sentiment d'appartenance à une même famille. Or, neuf ans après l'entrée en vigeur du Code, et donc de son article 32, il faut bien constater qu'on ne rencontre guère d'enfants éthiopiens nés dans cet intervalle et possédant un nom de famille; ceci est en contradiction formelle avec le prescrit du droit moderne tel qu'il a été voté par le Parlement, représentant la Nation, et promulgué par l'Empereur. Bien ou contraire, cet état de choses est considéré comme parfaitement normal par les Ethiopiens y compris ceux dont l'éducation d'une part et les fonctions qu'ils exercent de l'autre, devraient faire les témoins de la modernisation du pays et de son système juridique. C'est ainsi que j'ai eu l'occasion d'enseigner à des directeurs de ministère, voire à des ministres, à des magistrats, à des procureurs, à des officiers de police (y compris l'un des plus hauts représentants de la hiérarchie policière), à des parlementaires; tous, sans exception, admettaient avoir délibérément ignoré les forformalités légales relatives aux noms de famille dans les cas où ils avaient eu des enfants depuis 1960; quant à ceux pour qui cette possibilité appartenait encore à l'avenir, ils ne faisaient pas mystère de ce que serait leur attitude lorsqu'il leur faudrait considérer le respect du prescrit du Code: L'inobservance pure et simple. En outre, il est symptomatique de constater que les justifications fournies ne tenaient jamais à l'inadéquacité du système administratif du pays (les règles relatives à l'état civil ne sont pas encore en vigueur bien qu'il existe dans de nombreuses villes et en tout cas à Addis Abéba des registres tenus par la municipalité et dans lesquels sont enregistrés les naissances, mariages et décès); leur opposition était bien plus profonde et visait précisément le fait qu'il s'agissait là d'une innovation entièrement contraire à la tradition nationale. Au nom de celle-ci, ils estimaient devoir s'opposer à ce que le droit moderne comportait comme élément innovateur. Devant pareille situation, il est permis de se demander évidemment si la matière $\mathrm{du}$ nom constitue un élément suffisamment important du développement pour justifier sa "modernisation" par l'introduction du nom de famille. Je crois cependant que le problème dépasse celui de l'option entre le droit moderne et la tradition pour déboucher sur un plan autrement important: celui du respect de la loi dès lors que celle-ci est l'expression de la volonté nationale exprimée à travers les organes constitutionnellement reconnus à cet effet. La situation actuelle aboutit en effet à laisser les citoyens juges de la mesure dans laquelle la loi nationale doit gouverner leur vie quotidienne. C'est là une attitude extrêmement dangereuse d'abord en ce qu'il sera toujours difficile de faire le partage entre les cas où le refus de la loi est sans importance (comme dans le cas du nom) et ceux où il ne 
peut être toléré; ensuite parce que pareille attitude commence avec l'inobservance du Code civil et se termine par celle du Code pénal ou de la Constitution; enfin, et c'est là sans doute l'argument le plus important, parce que l'inobservance délibérée et non réprimée de la loi contribue à l'affaiblissement de la force obligatoire de celle-ci et risque donc de mettre en péril son efficacité comme outil du développement. Ce dernier point est d'autant plus grave lorsque c'est précisément la tradition qui est invoquée contre le droit moderne; à ce moment le principe même de la transformation de celui-ci par autre chose que l'évolution des esprits est niée. A mon sens, pareille situation ne devrait pas pouvoir se produire et l'alternative posée au gouvernement est simple; ou bien il assure par tous les moyens à sa disposition le respect de la loi ou bien il décrète la suspension de celle-ci en attendant qu'il se décide à la faire respecter. La réponse à la première branche de l'alternative sera souvent que la suspension des dispositions relatives à l'état civil entrâ̂ne nécessairement l'inapplicabilité du droit sur le nom de famille. Je ne crois pas que ce soit là un argument valable. En effet le gouvernement a la possibilité en d'innombrables autres instances (et notamment dans le cours des procédures administratives et judiciaires) de faire pression sur les individus pour qu'ils se conforment à la loi et j'ai la conviction qu'une campagne systématique en ce sens ne pourrait que porter les fruits. L'autre hypothèse est la suspension des textes en cause, voire leur modification par la procédure parlementaire (celle-ci pourrait d'ailleurs être déclenchée par une campagne systématique de l'exécutif montrant sans ambages que celui-ci a l'intention de faire respecter la loi). Quel que soit le résultat final, il aurait au moins l'avantage de montrer ou bien qu'il n'y a pas de lois, ou bien que celles-ci doivent être respectées.

\section{Conclusion}

Les problèmes soulevés dans les paragraphes qui précèdent peuvent paraître triviaux à ceux qui considèrent qu'une fois que le contenu du droit a été modernisé, le processus du développement juridique en est amorcé pour autant. C'est précisément contre cette assertion, par trop aisée et confortable pour la bonne conscience des experts préparant des codes "modernes" pour les pays en voie de développement qu'il convient de s'élever. En ne se souciant pas de l'intelligibilité du droit moderne, de sa diffusion et enfin des possibilités pratiques de sa mise en oeuvre, on contribue indiscutablement au phénomène qui fait si souvent la cible de sarcasmes: le "placage" de structures modernes sur un monde qui n'est pas prêt à les recevoir. Le développement juridique est une possibilité; mais comme toute oeuvre de développement, il faut non seulement s'en donner les cadres, mais aussi les moyens et une obstination sans cesse renouvelée dans l'intention de réussir. 